\title{
Michelzinho: Jogo sério para o ensino de habilidades emocionais em pessoas com autismo ou deficiência intelectual
}

\author{
Adilmar C. Dantas ${ }^{1}$, Sara L. de Melo ${ }^{1}$, Leandro A. Neves ${ }^{2}$, Taynara P. Milessi ${ }^{1}$ \\ Marcelo Z. do Nascimento ${ }^{1}$ \\ ${ }^{1}$ FACOM - Faculdade de Ciência da Computação \\ Universidade Federal de Uberlândia \\ Uberlândia, MG, Brasil \\ ${ }^{2}$ Departamento de Ciências de Computação e Estatıstica (DCCE) \\ Universidade Estadual Paulista (UNESP) \\ São José do Rio Preto-SP, Brasil \\ email\{akanehar, saraluziamelo, neves.leandro, taynarapmilessi, \\ marcelo.zanchetta\}@gmail.com
}

\begin{abstract}
This paper presents a "serious"class game to help teach emotional and social skills in people with autistic spectrum disorder (ASD) and intellectual disability. The application allows developing the skills to recognize and express the basic emotions: joy, sadness, anger, disgust, surprise and fear. Through the theory of facial action coding system and digital image processing techniques it was possible to detect facial expressions and classify them into one of the six basic emotions. Initial experiments were carried out to acquire initial data and to make improvements in the application before its definitive completion. In addition, the proposal was evaluated on the dataset of images $C k+$, obtaining an accuracy rate of $88.8 \%$ for the classification of basic emotions. Experiments with ASD carriers were conducted to evaluate the contributions and limitations of the proposed work.
\end{abstract}

Resumo. Esse trabalho apresenta um jogo da classe "sério"para auxiliar no ensino de competências emocionais e sociais em pessoas com Transtorno do Espectro Autista (TEA) e deficiência intelectual. A aplicação permite desenvolver as habilidades de reconhecer e expressar as emoções básicas: alegria, tristeza, raiva, desgosto, surpresa e medo. Por meio da teoria de sistema de codificação de ação facial e técnicas de processamento digital de imagens foi possível detectar expressões faciais e classifica-las em uma das seis emoções básicas. Experimentos iniciais foram realizados para a aquisição e dados iniciais e a realização de melhorias na aplicação antes de sua finalização definitiva. Essa proposta foi avaliada em imagens da base $C k+$, obtendo uma taxa de precisão de $88 \%$ para a classificação das emoções básicas. Experimentos com portadores do TEA foram realizados para avaliar as contribuições e limitações do trabalho proposto.

\section{Introdução}

O Transtorno do Espectro Autista (TEA) é considerado uma síndrome do neurodesenvolvimento que apresenta diferentes níveis de leve até grave. Esse transtorno pode ser 
VIII Congresso Brasileiro de Informática na Educação (CBIE 2019)

Anais do XXX Simpósio Brasileiro de Informática na Educação (SBIE 2019)

tratado como uma deficiência mental que compromete fatores cognitivos, em especial, as habilidades sociais e de comunicação [Mesibov et al. 2013]. De acordo com dados publicados pela Agência Americana do Centro de Controle e Prevenção de Doenças, estima-se que uma em cada 88 crianças são afetadas pelo TEA. Dados publicados pela Organização Mundial da Saúde (OMS), mostram que, um em cada 160 crianças tem sinais relacionados ao TEA [OMS 2017]. No Brasil, com uma população superior a 200 milhões de habitantes, estima-se que cerca de dois milhões pode ter sinais relacionados a esse transtorno [Control and Prevention 2018].

Uma das principais dificuldades enfrentadas pelos indivíduos com TEA está relacionada a capacidade de reconhecer e expressar as emoções da face de uma pessoa de forma natural. Essa restrição prejudica as interações sociais [Baron-Cohen et al. 2005]. Segundo Dawson et al. [Dawson et al. 2005], ter a capacidade de reconhecer e expressar as emoções é extremamente importante para as relações com outros indivíduos. Embora algumas pessoas com TEA, com alta funcionalidade, sejam capazes de entender as emoções por meio das expressões faciais, grande parte tem limitações no reconhecimento das expressões faciais presentes em cada emoção [Annaz et al. 2009, Elder et al. 2006].

Um outro grupo de pessoas que também é afetado pela dificuldade de representação das emoções são os portadoras de deficiência intelectual [Zaja and Rojahn 2008]. No trabalho de Wishart et al. [Wishart et al. 2007], fenótipos comportamentais de pessoas com síndrome de Williams e síndrome de Down foram investigadas e constataram essas limitações. De forma semelhante aos indivíduos com TEA, essas características podem comprometer uma série de características sóciocomportamentais. Isso mostra que trabalhos para o reconhecimento e expressão das emoções são importantes para pessoas em uma sociedade.

Uma das propostas para trabalhar essas restrições nestas pessoas é o Sistema de Comunicação de Intercâmbio de Fotos (SCIF) [Chien et al. 2015]. Essa solução é uma das técnicas pedagógicas mais utilizadas para auxiliar pessoas diagnosticadas com TEA, em especial, crianças para se adaptarem as relações sociais. O SCIF é uma ferramenta desenvolvida por especialistas em educação com a finalidade de complementar e desenvolver as habilidades de comunicação das crianças com outras pessoas [Bondy and Frost 1994].

Nos últimos anos, diversos estudos demonstraram que realizar intervenções em indivíduos com TEA com uso de tecnologias suportadas por computadores ou smartphone podem contribuir de forma mais efetiva no ensino de habilidades funcionais, sociais e comportamentais [Ayres and Langone 2005]. Nos trabalhos de [Chen et al. 2016, Silva et al. 2017], os autores apresentaram soluções inovadoras com uso de tecnologias para ajudar os indivíduos com TEA à desenvolverem e aprimorarem as competências emocionais e sociais. Essa área vem demandando cada vez mais novas pesquisas que possam contribuir com o aprimoramento de habilidades emocionais por meio de soluções que envolva novas tecnologias.

No ambiente educacional, tem se observado o desenvolvimento de ferramentas interativas como jogos para estimular habilidades de aprendizagem. Os jogos, denominados jogos sérios (do inglês, serious games - $S G$ ), pode ser definido como uma abordagem desenvolvida por meio dos princípios do design de jogo com objetivo de explorar conteúdos 
VIII Congresso Brasileiro de Informática na Educação (CBIE 2019)

Anais do XXX Simpósio Brasileiro de Informática na Educação (SBIE 2019)

educacionais ou treinamento [Charsky 2010]. Para os autores em [Dickey 2005], os SG usam as características de atividades de entretenimento para oferecer aos usuários experiências motivacionais para o aprendizado. Baseado neste contexto, esse trabalho apresenta um jogo que estimula as habilidades emocionais em um ambiente de entretenimento. O jogo denominado "Michelzinho" integra técnicas de processamento de imagem e inteligência artificial com objetivo de explorar e ensinar emoções para os indivíduos com TEA ou deficiência intelectual.

\section{Sistema de Comunicação de Intercâmbio de Fotos}

O Sistema de Comunicação de Intercâmbio de Fotos (SCIF) é composto por uma grande variedade de cartões com ilustrações. Cada cartão contém uma imagem e uma descrição que pode ser de um objeto, ação ou uma das emoções como exibido na Figura 1. Assim, a integração do SCIF permite que indivíduos construam frases e possam mostrar aos cuidadores e/ou tutores qual o estado emocional naquele momento.

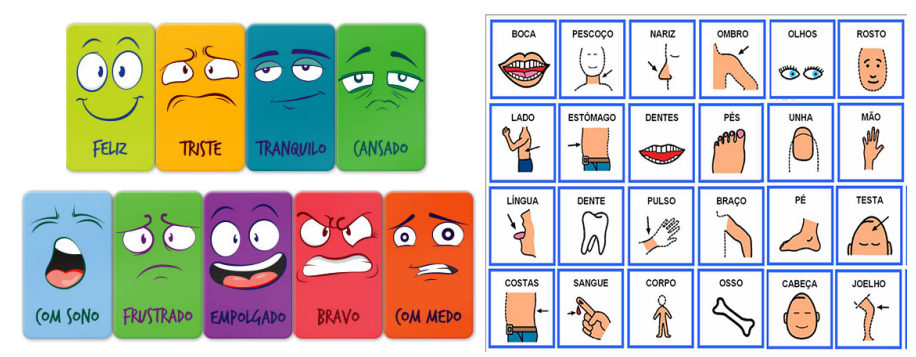

Figura 1. Cartões SCIF para o ensino das emoções e as partes do corpo humano.

A utilização do SCIF tem como principal característica não requer que os indivíduos possuam habilidades antes de usá-lo. As pessoas com TEA possuem habilidades cognitivas únicas devido ao grau de comunicação, assim o SCIF tem contribuído para o tratamento e no auxílio de cuidadores, membros da família e professores [Bondy and Frost 1994]. No entanto, apesar de ser uma solução muito empregada para interação entre os indivíduos e os cuidadores, essa solução encontra limitações, conforme descrito a seguir:

- Desenvolvimento e construção: Para a criação dos cartões com SCIF é necessário um trabalho manual realizado em uma série de etapas. Essas etapas envolvem a elaboração e a impressão dos cartões, recorte, plastificação e inserção de fixadores. Esse trabalho demanda tempo e recursos financeiros para criação do material.

- Portabilidade: Conforme os pacientes vão aprimorando suas habilidades cognitivas, os materiais elaborados não se tornam mais aplicáveis e são acumulados, o que torna necessário armazená-los em pastas, caixas, etc. Esse armazenamento exige espaço e precisa ser transportado sempre que necessário.

- Gerenciamento: Conforme os materiais são produzidos há um aumento da quantidade de informações geradas com os cartões. Isso dificulta o controle, no momento da intervenção, pois o responsável deve recuperar a informação do conjunto de cartões de cada um dos indivíduos. Esse problema pode criar um distância entre o especialista e o indivíduo no momento das sessões de intervenção. 
VIII Congresso Brasileiro de Informática na Educação (CBIE 2019)

Anais do XXX Simpósio Brasileiro de Informática na Educação (SBIE 2019)

\section{Metodologia}

Na Figura 2 é apresentado a estrutura do jogo com suas principais funcionalidades. Esse aplicativo possui quatro principais módulos: aplicação, processamento de imagens, banco de dados e inteligencia artificial. Todos esses módulos foram implementados em máquinas virtuais utilizando conceitos da computação em nuvem [Mell et al. 2011].

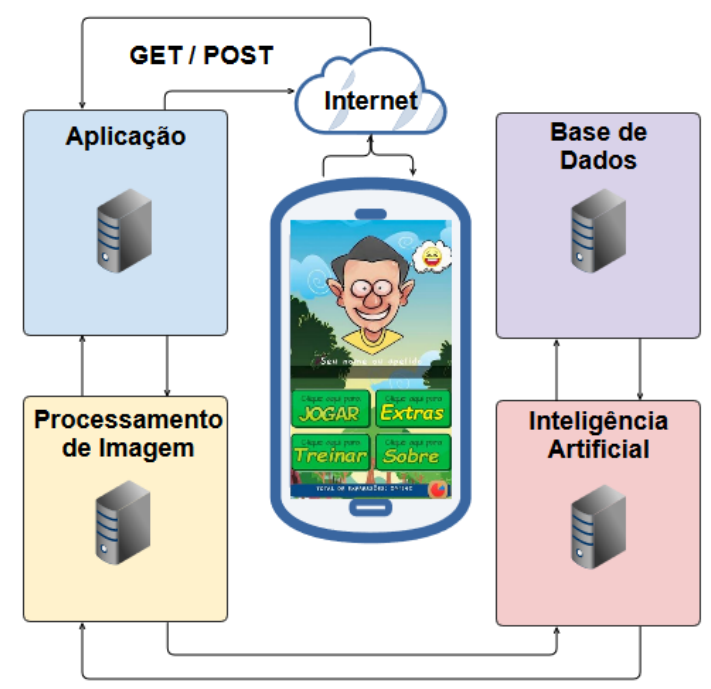

Figura 2. Estrutura do jogo com os principais módulos e fluxos de execução.

Neste trabalho, o módulo aplicação tem como função a comunicação por meio de uma interface entre o usuário e os outros módulos. Um exemplo é o controle de tela, acesso à câmera e informações aos usuários. Nessa etapa, também há uma interface digital da aplicação composta pelo: personagem "Michelzinho", cenários e elementos gráficos (botões, controles, etc).

Nesta fase, a modelagem e desenvolvimento da parte gráfica foi realizada com o software Blender. Esse software permite a criação de modelos em 3D, manipulação, animação, simulações, renderizações e rastreamento de movimento [Brito 2007]. Para representação das expressões faciais no personagem modelado foi utilizado o software Makehuman. Esse software open source que gera modelos de humanoides em 3D, com uma grande qualidade de detalhes e informações para a aplicação [Bagnara et al. 2018]. Na Figura 3 é apresentado o personagem em 3D com detalhes para as expressões faciais na representação de uma emoção.

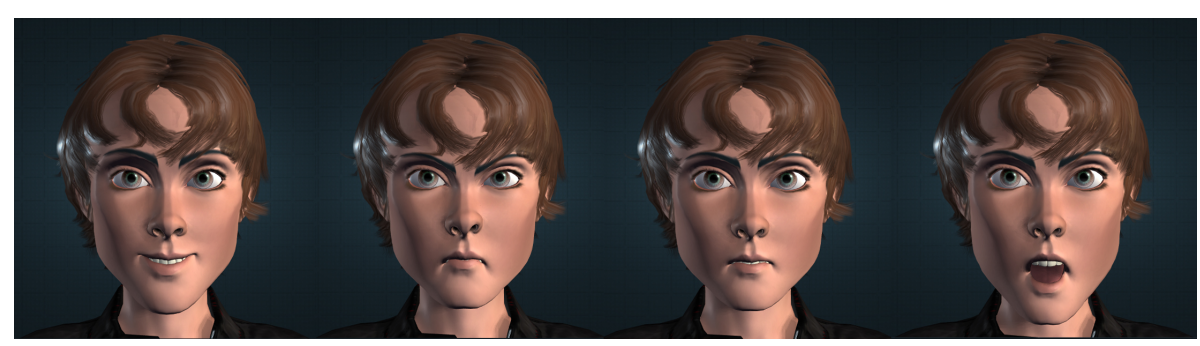

Figura 3. Personagem em 3D responsável por demonstrar as emoções por meio das expressões faciais. 
O módulo processamento de imagens é responsável pela manipulação das imagens da face do usuário dentro da aplicação. Nesta etapa, informações são extraídas da região da face por meio de métodos para detecção da face e extração de características.

Neste módulo, inicialmente, a face do usuário é capturada em imagens de vídeo com uma webcam. As imagens em modelo de cores RGB são convertidas para níveis de cinza. Esse processo é realizado com objetivo de eliminar possíveis ruídos presentes nos canais das imagens coloridas conforme relatos em [Lienhart and Maydt 2002].

Então, pontos relevantes da face devem ser detectados para a classificação da emoção. Nesta etapa, o algoritmo proposto por [Viola and Jones 2004] foi empregado com objetivo de fazer a detecção das regiões de interesse da face do usuário. A técnica de detecção da face é baseada no método proposto por Viola-Jones, que tem como característica analisar a aparência dos objetos e "aprender" as características mais relevantes em um conjunto de imagens. Esse procedimento pode ser usado em imagens estáticas ou vídeos capturados em tempo real.

Após detectar as regiões de interesse na face do usuário, o histograma de níveis de cinza foi calculado e 68 pontos foram detectados com base em marcadores obtidos da base CK+ [Kanade and Cohn 2005]. Na Figura 4 é mostrado os 68 pontos obtidos da face do usuário. Dentre os pontos, apenas 44 pontos foram selecionados de acordo com as Unidades de Ação (UA) propostas na teoria FACS [Ekman and Friesen 1978]. O uso das UA como características extraídas da face permitiu obter um vetor de características a ser analisado na etapa de predição e teste da classificação das emoções.

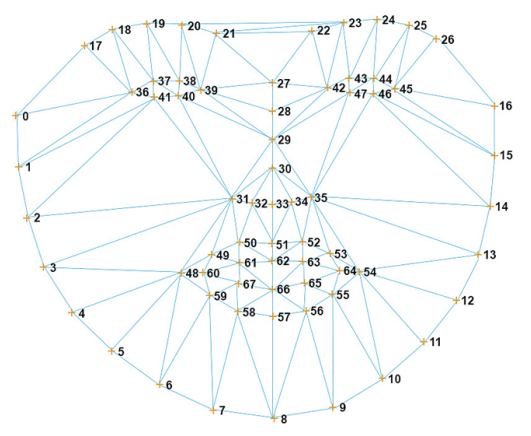

Figura 4. Marcadores capturados na imagem do rosto do usuário.

Além disso, o algoritmo proposto por [Fleet and Weiss 2006] baseado em fluxo óptico foi implementado com objetivo de predizer o movimento dos músculos da face em sequências de imagens durante o movimento do usuário. Com as regiões localizadas, as informações foram extraídas e enviadas ao módulo responsável pela classificação das emoções. O módulo Inteligência Artificial é utilizado para a classificação das emoções por meio de algoritmos de classificação. Nesta etapa, foram empregados o algoritmo de classificação baseado em Árvore de Decisão (AD) [Dantas et al. 2015] e uma Rede Neural Convolucional (RNC).

No algoritmo AD, na etapa de treinamento do modelo foi utilizado as imagens da base [Kanade and Cohn 2005]. Essa base foi escolhida para treinamento devido ao fato que possui imagens de diferentes etnias e sexo e as representações do estado de cada uma das emoções. Nesta etapa, foram usadas as 1452 imagens em escala de cinza para 
VIII Congresso Brasileiro de Informática na Educação (CBIE 2019)

Anais do XXX Simpósio Brasileiro de Informática na Educação (SBIE 2019)

treinamento de cada emoção. Então, com a predição do classificador, os vetores com as informações obtidas da fase de processamento de imagem são empregados como entrada para a fase de teste e classificação.

A RNC, com aprendizagem profunda, foi construída usando a biblioteca TFLearn por meio do Tensorflow, um framework de aprendizado de máquina desenvolvido pelo Google para criar, projetar, construir e treinar modelos de aprendizagem profunda [Google 2019] por meio da linguagem Python. A RNC desenvolvida foi baseada no trabalho de [Gudi 2015]. A rede original começa com uma camada de entrada de 48 por 48, correspondendo ao tamanho dos dados de entrada. Esta camada é seguida por uma camada convolucional, uma camada de normalização de contraste local, e uma camada de max pooling, respectivamente. A rede termina com mais duas camadas convolucionais e uma camada ligada ao softmax da camada de saída, o Dropout foi aplicado em todas as camadas conectadas e o ReLu em todas as camadas. A camada max pooling tem como função reduzir o número de parâmetros e consequentemente o custo computacional necessário para treinar o modelo. Na Figura 5 é exibida arquitetura da rede.

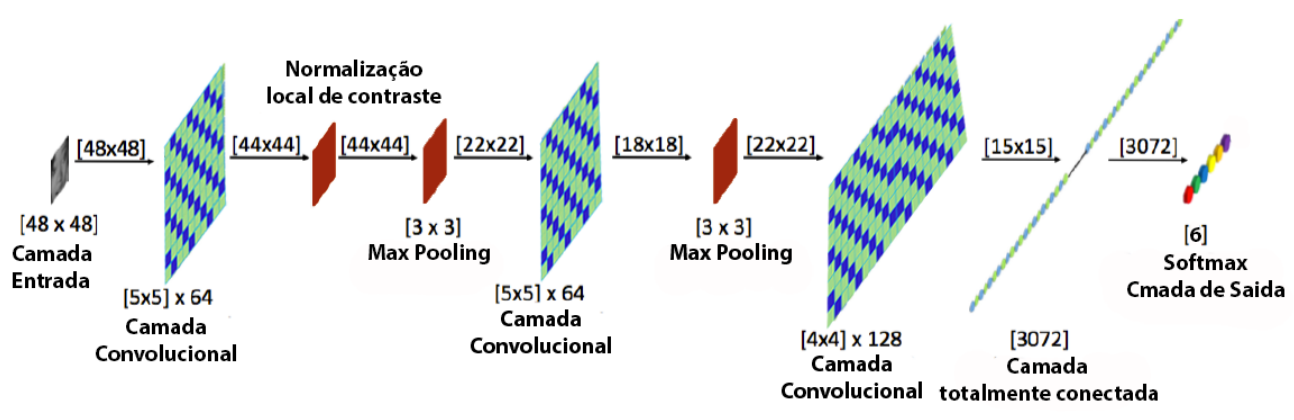

Figura 5. Visão geral da arquitetura da rede implementada.

Para avaliação dos algoritmos foi empregado as métricas estatísticas como: recall, que pode ser entendido como uma medida para avaliar a capacidade de um modelo encontrar os dados relevantes dentro de conjunto de dados. Também foi empregado a precisão, que pode ser definida como o número de verdadeiros positivos dividido pelo número de positivos verdadeiros mais o número de falsos positivos e, por fim, a avaliação $F 1$-score definida pela equação 1 .

$$
F_{1}=\frac{\text { Precission } \times \text { Recall }}{\text { Precission }+ \text { Recall }}
$$

A medida F1 Score é necessária quando se deseja buscar um equilíbrio entre precisão e recuperação se adaptando bem ao solução proposta assim como em outros trabalhos da área [Valstar and Pantic 2012, Valstar et al. 2017, Acevedo et al. 2017].

Para armazenar as informações obtidas com resultado das classificações e realizar possíveis análise, há também um módulo banco de dados que armazena as métricas dos resultados de cada usuário. O banco de dados foi construído em Structured Query Language. Essa linguagem é utilizada por diversas empresas de tecnologia como Google e Facebook [MySql 2018]. 
VIII Congresso Brasileiro de Informática na Educação (CBIE 2019)

Anais do XXX Simpósio Brasileiro de Informática na Educação (SBIE 2019)

\section{Resultados Experimentais}

Nessa seção são apresentados os resultados obtidos para os experimentos realizados neste estudo. Essa seção foi divida em duas partes, uma para os resultados obtidos a partir de experimentos realizados para avaliar as técnicas de classificação de emoções e outra para os resultados da aplicação do jogo.

\subsection{Avaliação das Técnicas para Classificação das Emoções}

O método proposto para as duas abordagens de classificação foram avaliadas em um conjunto de imagens da base $\mathrm{Ck}+$ [Kanade and Cohn 2005]. Essa base é dividida em imagens de pessoas demonstrando as emoções: alegria (324 imagens), tristeza (253 imagens), raiva (183 imagens), surpresa (328 imagens), desgosto (182 imagens) e medo (182 imagens). Na Tabela 1 são apresentados os valores das métricas quantitativas obtidas para os algoritmos de classificação empregados nesta proposta.

\begin{tabular}{|c|c|c|c|c|c|c|}
\hline Emoção & $\begin{array}{c}\text { Precisão } \\
\text { (AD) }\end{array}$ & $\begin{array}{c}\text { Recall } \\
\text { (AD) }\end{array}$ & $\begin{array}{c}\text { F1-Score } \\
\text { (AD) }\end{array}$ & $\begin{array}{c}\text { Precisão } \\
\text { (RNC) }\end{array}$ & $\begin{array}{c}\text { Recall } \\
\text { (RNC) }\end{array}$ & $\begin{array}{c}\text { F1-Score } \\
\text { (RNC) }\end{array}$ \\
\hline Alegria & 1.00 & 1.00 & 1.00 & 0.90 & 0.9 & 0.95 \\
\hline Tristeza & 0.85 & 0.85 & 0.92 & 0.28 & 0.28 & 0.43 \\
\hline Raiva & 0.88 & 0.88 & 0.93 & 0.50 & 0.50 & 0.66 \\
\hline Desgosto & 0.88 & 0.88 & 0.93 & 0.62 & 0.62 & 0.76 \\
\hline Surpresa & 0.91 & 0.91 & 0.95 & 0.77 & 0.77 & 0.87 \\
\hline Medo & 0.70 & 0.70 & 0.82 & 0.36 & 0.36 & 0.53 \\
\hline Média & 0.88 & 0.87 & 0.92 & 0.57 & 0.57 & 0.70 \\
\hline
\end{tabular}

Tabela 1. Resultados obtidos a partir das métricas para os classificadores analisadas.

Observando os dados apresentados na Tabela 1 é possível notar que os valores de precisão foram satisfatórios para grande parte das emoções. Apenas com as emoções desgosto e medo, os classificadores não tiveram resultados expressivos. Os valores ocorrem porque as emoções medo e desgosto possuem muitas UA semelhantes com outras emoções, o que dificulta a classificação, conforme já relatado no trabalho de [Dantas et al. 2015]. De forma geral, os resultados médios foram significativos com um valor de precisão de 0.88 para a classificação com o algoritmo de [Dantas et al. 2015], mas não representativo para o uso da RNC com um valor de 0.57.

Os valores obtidos por meio da métrica Recall com a proposta de [Dantas et al. 2015] se mostraram satisfatórias com um valor médio de 0.87, o que mostra que o modelo conseguiu detectar de forma correta um número significativo de casos da base investigada. A medida $F 1$ - Scores é a média ponderada de Precisão e Recall. Portanto, essa pontuação considera os falso-positivos e os falso-negativos. Intuitivamente, o F1 - Score é geralmente mais importante que a precisão, especialmente quando se trabalha com amostras de classes desiguais. Nos testes realizados com AD, os resultados foi de 0.92 o que é significativo para classificação das emoções. Como acontece com o banco de imagens utilizado, que possuiu um número não balanceado de amostras para as emoções básicas. 
VIII Congresso Brasileiro de Informática na Educação (CBIE 2019)

Anais do XXX Simpósio Brasileiro de Informática na Educação (SBIE 2019)

\subsubsection{Avaliação inicial do jogo}

Para avaliar a aplicação dos algoritmos propostos, foi desenvolvido um total de 50 fases temáticas para que os usuários pudessem reconhecer ou expressar umas das emoções básicas por meio dos seus dispositivos móveis. As fases foram desenvolvidas com elementos e dinâmica de jogo para possibilitar atrair a atenção dos usuários, conforme exibido na Figura 6.
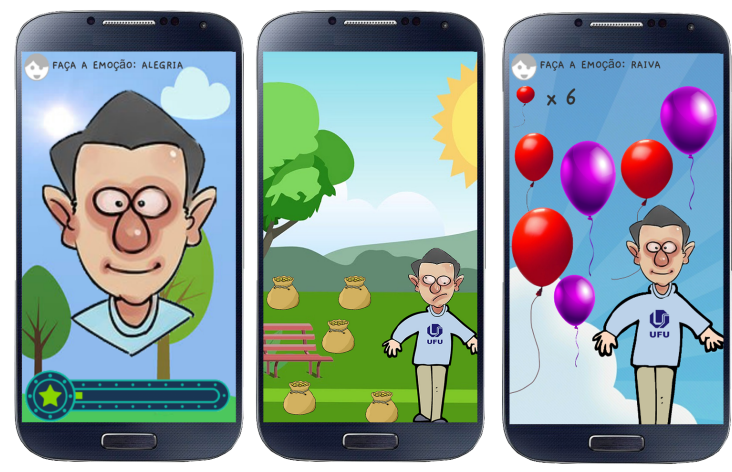

Figura 6. Exemplo de fases desenvolvidas para o jogo.

A aplicação dos experimentos e investigação com usuários foi devidamente submetida ao comitê de ética da Universidade Federal de Uberlândia para aprovação e aplicação dentro das normas vigentes para as pesquisas que envolvem seres humanos, com o número 82555417.0.0000.5152. Essa ferramenta foi disponibilizado de forma gratuita na loja de aplicativos Google Play para Android. No período de seis meses informações dos usuários, partidas jogadas e melhorias da ferramenta foram coletadas. Nesse período foram realizado mais de 250 mil partidas jogadas por mais de 2 mil jogadores.

\section{Considerações Finais}

Esse trabalho apresentou uma ferramenta do tipo SG capaz de contribuir para o ensino de habilidade emocionais em pacientes com TEA ou deficiência intelectual. Neste caso, tem-se a combinação de tecnologias computacionais, teorias educacionais e fundamentos psicológicos para pessoas com TEA ou deficiência intelectual. A aplicação foi capaz de contribuir para a solução de alguns problemas apresentados por métodos tradicionais descritores pelos especialistas da área como, por exemplo, o sistema PECS.

O emprego do algoritmo de classificação proposto no estudo de [Dantas et al. 2015] mostrou-se mais relevante em termos de valores médios para medidas de desempenho. Isso foi observado com a métrica precisão com valor de 0.88 e medida $F 1-$ Score de 0.92. Uma das limitações apresentadas pelos usuários é a necessidade de um dispositivo móvel e a falta da ferramenta disponibilizada em outros idiomas. Como trabalhos futuros, será investigado a aplicação do jogo com um grupo de controle com indivíduos com TEA para extrair mais informações das contribuições. Além disso, também será investigado a possibilidade de desenvolver o jogo para outros plataformas e traduzir as informações de mídia para outros idiomas. 
VIII Congresso Brasileiro de Informática na Educação (CBIE 2019)

Anais do XXX Simpósio Brasileiro de Informática na Educação (SBIE 2019)

\section{Referências}

Acevedo, D., Negri, P., Buemi, M. E., Fernández, F. G., and Mejail, M. (2017). A simple geometric-based descriptor for facial expression recognition. In Automatic Face \& Gesture Recognition (FG 2017), 2017 12th IEEE International Conference on, pages 802-808. IEEE.

Annaz, D., Karmiloff-Smith, A., Johnson, M. H., and Thomas, M. S. (2009). A crosssyndrome study of the development of holistic face recognition in children with autism, down syndrome, and williams syndrome. Journal of experimental child psychology, 102(4):456-486.

Ayres, K. M. and Langone, J. (2005). Intervention and instruction with video for students with autism: A review of the literature. Education and Training in Developmental Disabilities, 40(2):183-196. Cited By :93.

Bagnara, S., Tartaglia, R., Albolino, S., Alexander, T., and Fujita, Y. (2018). Proceedings of the 20th Congress of the International Ergonomics Association (IEA 2018): Volume II: Safety and Health, Slips, Trips and Falls, volume 819. Springer.

Baron-Cohen, S., Wheelwright, S., Lawson, J., Griffin, R., Ashwin, C., Billington, J., and Chakrabarti, B. (2005). Empathizing and systemizing in autism spectrum conditions. Handbook of autism and pervasive developmental disorders, 1:628-639.

Bondy, A. S. and Frost, L. A. (1994). The picture exchange communication system. Focus on autistic behavior, 9(3):1-19.

Brito, A. (2007). Blender 3D-Guia do Usuário. Novatec Editora.

Charsky, D. (2010). From edutainment to serious games: A change in the use of game characteristics. Games and culture, 5(2):177-198.

Chen, C.-H., Lee, I.-J., and Lin, L.-Y. (2016). Augmented reality-based video-modeling storybook of nonverbal facial cues for children with autism spectrum disorder to improve their perceptions and judgments of facial expressions and emotions. Computers in Human Behavior, 55:477-485.

Chien, M.-E., Jheng, C.-M., Lin, N.-M., Tang, H.-H., Taele, P., Tseng, W.-S., and Chen, M. Y. (2015). ican: A tablet-based pedagogical system for improving communication skills of children with autism. International Journal of Human-Computer Studies, 73:79-90.

Control, C. D. and Prevention (2018). Autism spectrum disorder (asd)@ONLINE.

Dantas, A. C., de Melo, S., Moura, F., Fernandes, M., Gerais-Brasil, U.-M., and GeraisBrasil, B.-M. (2015). Reconhecimento dinâmico de emoções através de expressões faciais utilizando árvore de decisão. In Anais do Simpósio Brasileiro de Informática na Educação, volume 26, page 1102.

Dawson, G., Webb, S. J., and McPartland, J. (2005). Understanding the nature of face processing impairment in autism: insights from behavioral and electrophysiological studies. Developmental neuropsychology, 27(3):403-424.

Dickey, M. D. (2005). Engaging by design: How engagement strategies in popular computer and video games can inform instructional design. Educational technology research and development, 53(2):67-83. 
VIII Congresso Brasileiro de Informática na Educação (CBIE 2019)

Anais do XXX Simpósio Brasileiro de Informática na Educação (SBIE 2019)

Ekman, P. and Friesen, W. (1978). Facial action coding system. Alto: Consulting Psychologist Press.

Elder, L. M., Caterino, L. C., Chao, J., Shacknai, D., and De Simone, G. (2006). The efficacy of social skills treatment for children with asperger syndrome. Education and Treatment of Children, pages 635-663.

Fleet, D. and Weiss, Y. (2006). Optical flow estimation. In Handbook of mathematical models in computer vision, pages 237-257. Springer.

Google (2019). An end-to-end open source machine learning platform @ONLINE.

Gudi, A. (2015). Recognizing semantic features in faces using deep learning. arXiv preprint arXiv:1512.00743.

Kanade, T. C. and Cohn, J. (2005). Au-coded facial expression database.

Lienhart, R. and Maydt, J. (2002). An extended set of haar-like features for rapid object detection. In Image Processing. 2002. Proceedings. 2002 International Conference on, volume 1, pages I-I. IEEE.

Mell, P., Grance, T., et al. (2011). The nist definition of cloud computing.

Mesibov, G. B., Adams, L. W., and Klinger, L. G. (2013). Autism: Understanding the disorder. Springer Science \& Business Media.

MySql (2018). Why mysql@ONLINE.

OMS (2017). Autism spectrum disorders, key facts@ ONLINE.

Silva, M., Soares, A., and Benitez, P. (2017). Ambiente digital para ensino e acompanhamento personalizado de estudantes com autismo: proposta com uso de dispositivos móveis. In Brazilian Symposium on Computers in Education (Simpósio Brasileiro de Informática na Educação-SBIE), volume 28, page 1047.

Valstar, M. F. and Pantic, M. (2012). Fully automatic recognition of the temporal phases of facial actions. IEEE Transactions on Systems, Man, and Cybernetics, Part B (Cybernetics), 42(1):28-43.

Valstar, M. F., Sánchez-Lozano, E., Cohn, J. F., Jeni, L. A., Girard, J. M., Zhang, Z., Yin, L., and Pantic, M. (2017). Fera 2017-addressing head pose in the third facial expression recognition and analysis challenge. In 2017 12th IEEE International Conference on Automatic Face \& Gesture Recognition (FG 2017), pages 839-847. IEEE.

Viola, P. and Jones, M. J. (2004). Robust real-time face detection. Int. J. Comput. Vision, 57(2):137-154.

Wishart, J., Cebula, K., Willis, D., and Pitcairn, T. (2007). Understanding of facial expressions of emotion by children with intellectual disabilities of differing aetiology. Journal of Intellectual Disability Research, 51(7):551-563.

Zaja, R. H. and Rojahn, J. (2008). Facial emotion recognition in intellectual disabilities. Current Opinion in Psychiatry, 21(5):441-444. 